\title{
Influence of familiarization on preschool children's motor tests results
}

\author{
Bojan Međedović*, Romana Romanov, Violeta Zubanov, Dušan Perić, Dušan Stupar, \\ and Zlatko Ahmetović
}

Faculty of Sport and Tourism, Educons University, Novi Sad, Serbia

Copyright: () 2018 B. Međedović et al. This is an open access article licensed under the Creative Commons Attribution License (http://creativecommons.org/licenses/by/4.0/).

\begin{abstract}
Background: Various field tests can be used to evaluate children's motor skills. Because of the complexity of their motor skills, which at their age are still in a very intensive stage of development, it is inadequate to use the same test protocol as in the adult population, as children receive and process information in a different way. Some evidence showed that familiarization with test protocols is important in children's motor skills evaluation process. Objective: The purpose of this study was to examine the influence of familiarization on motor test results for the assessment of motor skills in preschool children population. A controlled research study design was conducted. Methods: The sample included 40 children, 20 boys and 20 girls (age $5.89 \pm 0.42$ years). Subjects underwent two testing sessions with two weeks between the trials, performed 6 motor skills: leg power, flexibility, abdominal muscles endurance, shoulder muscles endurance, agility, and running speed. Before the second testing session, subjects underwent a period of familiarization with motor tasks in 3 sessions, with 3 trials every 3 days. Results: Significantly better results in the second testing session were observed in countermovement jump (with both fixed and free arms), curl-ups, speed and agility test. The present study indicates that children significantly improved the results in the motor test of explosive strength, muscle endurance, speed and agility, being influenced by familiarization. Conclusions: The results of this study indicate that familiarization with the test procedures prior to testing affect the results of the motor test, and represent an important part of the evaluation of motor skills of preschool children.
\end{abstract}

Keywords: motor test, evaluation, vertical jump, flexibility, endurance, agility

\section{Introduction}

Improvement of motor skills is an important segment of a child's entire development; therefore, the studies on children's development refer to assessing skills in the early stages of development. Motor development is the process of acquiring movement patterns and skills. Motor skills are determined by accuracy, precision and economy of movement performance (Malina \& Bouchard, 1991). Various field tests can be used to establish children's motor skill proficiency. Thus, it is important to consider prescribed test protocol. It should also be mentioned that at the preschool age, a great variability of results can occur between day-to-day measurements, but also between attempts within one single day (Malina \& Bouchard, 1991). Not all motor skills are developed on the same level at the preschool

\footnotetext{
* Address for correspondence: Bojan Međedović, Faculty of Sport and Tourism, Educons University, Radnicka 30a, Novi Sad 21000, Serbia. E-mail: bojan.medjedovic@tims.edu.rs
}

age. Immaturity of neuromuscular system and body size and composition, rather than weak motor coordination, is a limiting factor in performing a certain movement (Malina \& Bouchard, 1991). Specific skills can be developed through movement repetition only if a relevant level of motor maturity has been attained (American Academy of Pediatrics, 1992). Motor skill development and learning influence the entire growth and development of the children (Barnett, van Beurden, Morgan, Brooks, \& Beard, 2008; Fisher et al., 2005).

During early childhood motor skills are still in a very intensive stage of development. Because of complexity of children's motor skills, it is very difficult to find research result in which those motor dimensions are completely isolated as in the adult population, and researchers using the same protocols encounter some problems and suggest new methods and standards and tests that could better identify the motor status of a child (Tomac, Hraski, \& Sporis, 2012).

Motor development should have the role of a control parameter in a child's entire development 
(Bushnell \& Boudreau, 1993; Yoon, Scott, Hill, Levitt, \& Lambert, 2006), therefore, there is a need for testing protocols for collecting and evaluation real indicators of a child's motor status. Studies on children's development refer to assessment skills in the early stages of development with the aim of investigation in the sense of learning new movement structures and skill proficiency (Gwynne \& Blick, 2004; Piek, Dawson, Smith, \& Gasson, 2008).

For this reason, Burton and Miller (1998) identify five main reasons for testing: identification and categorisation, planning of medical treatment or activity, tracking changes within a certain period, giving feedback information to the person who exercises, as well as prediction of improvement.

During the process of development and improvement their motor abilities, children often use feedback information differently from adults (Sullivan, Kantak, \& Burtner, 2008). It has been confirmed before (Bala, 1999; Sullivan et al., 2008) that during the performance of motor skills and adoption of new motor skills for children, using the adult testing protocols is inadequate, as children receive and process information in a different way. Bala (1999) also found that the results of second and third measurement show the greatest values, except for the endurance assessment tests, and concluded that children learn to perform the movement the first time they perform it. The author suggested necessary modifications of tests and testing methods, recommending measuring in several attempts, so as to allow the children to understand the exercise and prepare to perform it. The author concluded that children should be provided with adequate instructions, have their motivation increased and allow for several repetitions of each test.

On a daily basis, children engage in motor activity that leads to the progressive development of motor skills. Some of this activity leads to skill in functional tasks such as running, jumping, kicking, and throwing (Sullivan et al., 2008). Some of the previous studies that analysed the influence of familiarization on the running and jumping test results of the adults has shown different conclusions. Glaister et al. (2010) has shown a positive impact of familiarization on multiple running speed test result, while others (Moir, Button, Glaister, \& Stone, 2004; Moir, Sanders, Button, \& Glaister, 2005) find that process of familiarization did not improve jumping and running test results.

Evaluation of motor skills and monitoring of their development is a complex process, especially among children. This requires a high level of precision, reliability and objectiveness. The results of motor tests in preschool children are affected by several factors. The quality of demonstration is important, as well as the impact of motivation, and the adaptability of the individual child on the overall situation in which the measurements are being conducted (Tomac \& Hraski, 2016). Also, authors generally agree that it is necessary to prevent systematic measurement errors so as to achieve high test reliability. The learning effect can cause systematic error in the tests for the assessment of motor skills, as it reduces the reliability of the measuring instrument. The familiarization process or previous experience with the measuring instrument can overcome the effect of learning and improve the reliability of measurements, and high correlations between the series of measurements (Hopkins, 2000; Patterson, Bennington, \& De La Rosa, 2001; Thomas \& Nelson, 1996).

Taking into account the problems noted during testing and the fact that those problems are more prominent in testing children, it is necessary to establish the impact of familiarization on the final result of each test. Thus, the purpose of this study was to examine the impact of familiarization on motor tests results for the assessment of motor skills in preschool children, such as its role in informing about movement components that can contribute to result improvement.

\section{Methods}

\section{Research design}

The research included preschool children from several preschool institutions, and testing was conducted during physical education classes in the morning. The testing consisted of two measurements: initial and final. Before, but also during the initial measurement, the clear information on the way of each test would be conducted was given. After that, the examinees were included in the process of familiarization with the testing procedures providing information on the quality of movement techniques. Due to limited time for testing, which include warm up and cool down activities, preschool children took each individual test three times. As children in that age grow and develop quickly (Malina \& Bouchard, 1991), the entire testing programme lasted for two weeks, leaving out the impact of biological maturation on the end result. To avoid forgetting of testing movements, sessions were organized every 3 days. Thus, every examinee performed 3 repetitions of each task every 3 days, with a total of 9 repetitions of each task with instruction. After that they had final testing, with clear instructions on proper test execution technique, as well as on the result achieved. All tests were preceded by a 10-minute warm-up, with the use of movements similar to those in testing procedures.

The research design was approved by the Ethics Committee at the Faculty of Sport and Tourism 
(Educons University) and conformed to the recommendations from the Declaration of Helsinki. All parents provided written informed consent. All parents agreed to their children taking part in the study.

\section{Participants}

The sample included 40 children, 20 boys and 20 girls (age $5.89 \pm 0.42$ years). Children who participated in this study attended a regular physical education program and were not involved in any further sports activities and had no previous experience with motor tests. All the children during the study were healthy and fully aware of the content measurements.

\section{Procedures}

In order to evaluate motor skills, the European Tests of Physical Fitness (Council of Europe, 1988) and American College of Sport Medicine (2010) test battery procedures were used and the following categories were measured: leg power, speed, agility, shoulder and abdominal muscle repetitive strength, and flexibility of lower back and hamstring muscles.

In order to estimate leg power, Squat jump, Countermovement jump with both fixed and free arms were estimated using contact mat (Axon Jump, Cordoba, Argentina). Countermovement jump requires the subject to begin in an upright posture with their feet shoulder width apart and with hands on the hips (fixed arms) and arms allowed to swing (free arms). After a brief downward phase (semi squat position) subjects jump straight up in the air with an all-out effort, maintaining hands on the hips to prevent arms from contributing to the jump and landing with extended legs on both feet at the same time. Squat jump requires the subject to begin in semi squat position with their feet shoulder width apart and with hands on the hips and then jump straight up. Three attempts for each jump type were allowed with the highest value included in further analysis. Jump height was calculated from the time the subject was off the mat by the computer which was connected to the platform.

In order to estimate the flexibility of lower back and hamstring area muscles, a Sit-and-reach test was used. The test was repeated three times and the farthestreached point (in centimetres) was recorded. In order to estimate the abdominal muscle repetitive strength, a curl-up test was used. The number of properly executed crunches in one minute is recorded as a final result. In order to estimate the shoulder muscle repetitive strength, push-ups were used. The number of properly executed push-ups, or to failure, was recorded as result. In order to estimate agility, a $10 \times 5$-meter running test was used (Eston \& Reilly, 2001). Test duration was recorded. Speed was measured using a 5-meter running test. The test was repeated two times, with a one-minute recovery between repetitions and the better time of the two tests was taken as a final result in this test. All tests were preceded by 10-minute warmups and movements used resembled those in testing procedures.

\section{Statistical analysis}

The data are expressed as Means \pm SD. The normality of the data distribution was tested by the Shapiro-Wilk test. Reliability was calculated using ICC coefficient. Paired samples $t$-test was used to establish the differences in means of the variables. $p$-values smaller than .05 were considered statistically significant. Effect size (Cohen's $d$ ) values between 0 to 0.3 represent a small effect size, between 0.3 and 0.6 moderate effect size, and more than 0.6 large effect size. For all calculations, statistics software SPSS was used (Version 17 for Windows for Windows; SPSS, Chicago, IL, USA).

\section{Results}

The Shapiro-Wilks test showed that the distribution does not deviate significantly from the normal distribution. The ICC coefficient between the series of measurements was found to be good at .644 . The results showed fair to high test-retest reliability: Squat jump ICC $=.456$, Countermovement jump with fixed arms ICC $=.654$, Countermovement jump with free arms ICC $=.617$, Curl-ups ICC $=.644$, Sit-and-reach ICC $=.845$, Running speed ICC $=.171$, Agility ICC $=.389$.

The main descriptive indicators are shown in Table 1. Significant difference between the initial and final measurements was observed in Countermovement jump, Countermovement jump with free arms, Curl-ups, Running speed, and Agility.

The results presented in Table 1 showed that familiarization had significant effect on tests scores in Countermovement jump from $15.18 \mathrm{~cm}$ to 17.64 $\mathrm{cm}$ (16.4\%); Countermovement jump with free arms from $16.47 \mathrm{~cm}$ to $20.14 \mathrm{~cm}$ (22\%); Curl-up from 11.72 to 18.82 repetitions $(60 \%)$; Running speed from 2.26 to $1.95 \mathrm{~s}(15.89 \%)$; and Agility from 26.59 to $25.15 \mathrm{~s}$ $(5 \%)$. Familiarization had no significant effect on tests scores in Squat jump where improvement was from 14.83 to $15.49 \mathrm{~cm}(4 \%), p=.30$; and Sit-and-reach test, where examinees showed worst score in final measure, from 29.37 to $28.75 \mathrm{~cm} \mathrm{(2 \% ),} p=.48$.

During the push-up test there was an error during measurement itself, and this parameter will no longer be used in this research. In that test there was a different administrator during initial and final measurement, and examinees were given different instructions of correct movement technique. 
Table 1

The main descriptive indicators, differences, and effect size

\begin{tabular}{|c|c|c|c|c|c|c|c|c|}
\hline & \multicolumn{2}{|c|}{ Initial measurement } & \multicolumn{2}{|c|}{ Final measurement } & \multirow[b]{2}{*}{$t(39)$} & \multirow[b]{2}{*}{$p$} & \multirow[b]{2}{*}{$95 \% \mathrm{CI}$} & \multirow[b]{2}{*}{ Cohen's $d$} \\
\hline & $M \pm S D$ & Range & $M \pm S D$ & Range & & & & \\
\hline SJ & $14.83 \pm 3.60$ & $4.9-20.4$ & $15.49 \pm 3.24$ & $7.0-22.0$ & -1.03 & .308 & {$[-1.97,0.64]$} & 0.19 \\
\hline $\mathrm{CJ}_{\mathrm{FI}}$ & $15.18 \pm 3.01$ & $7.1-20.4$ & $17.64 \pm 2.69$ & $13.0-23.8$ & -5.37 & $<.001$ & {$[-3.38,-1.53]$} & 0.86 \\
\hline $\mathrm{CJ}_{\mathrm{FR}}$ & $16.47 \pm 3.23$ & $9.0-22.8$ & $20.14 \pm 3.43$ & $13.2-28.3$ & -6.63 & $<.001$ & {$[-4.80,-2.55]$} & 1.10 \\
\hline $\mathrm{Pu}$ & $13.92 \pm 7.58$ & $1-32$ & $6.15 \pm 3.12$ & $1-14$ & 6.89 & $<.001$ & {$[5.49,10.06]$} & 2.45 \\
\hline $\mathrm{Cu}$ & $11.72 \pm 5.67$ & $2-23$ & $18.82 \pm 7.84$ & $6-37$ & -6.40 & $<.001$ & {$[-9.34,-4.86]$} & 1.04 \\
\hline $\mathrm{Si}$ & $29.37 \pm 7.88$ & $15-46$ & $28.75 \pm 7.39$ & $12-41$ & 0.70 & .488 & {$[-1.17,2.41]$} & 0.08 \\
\hline $\mathrm{Sp}$ & $2.26 \pm 0.28$ & $1.69-2.86$ & $1.95 \pm 0.29$ & $1.18-2.57$ & 5.06 & $<.001$ & {$[0.19,0.43]$} & 1.09 \\
\hline $\mathrm{Ag}$ & $26.84 \pm 2.42$ & $22.00-36.78$ & $25.15 \pm 2.27$ & $21.00-30.13$ & 3.15 & .003 & {$[0.52,2.37]$} & 0.72 \\
\hline
\end{tabular}

Note. $\quad \mathrm{CI}=$ confidence interval; $\mathrm{SJ}=$ squat jump $(\mathrm{cm}) ; \mathrm{CJ}_{\mathrm{FI}}=$ countermovement jump with fixed arms $(\mathrm{cm}) ; \mathrm{CJ}_{\mathrm{FR}}=$ countermovement jump with free arms (cm); $\mathrm{Pu}=$ push-ups (repetitions); $\mathrm{Cu}=$ curl-ups (repetitions); $\mathrm{Si}=$ sit-and-reach $(\mathrm{cm}) ; \mathrm{Sp}=\operatorname{running}$ speed (s); $\mathrm{Ag}=\operatorname{agility}(\mathrm{s})$.

\section{Discussion}

It has been agreed before that evaluation of motor skills of children should not be approached in the same manner as adult testing, because children process information in a different way, which could be a limiting factor in achieving maximal results in certain tests and requirements.

This study was conducted with the aim to examine the impact of familiarization on motor tests results for the assessment of motor skills in preschool children. The results indicate that familiarization significantly improves scores in several motor tests.

In the research of Tomac and Hraski (2016) results showed that children need more than three series for a familiarization with the test and for a stabilization of the results, as it was observed from the test performances of explosive strength, whereby there were no statistically significant differences except between the fourth and sixth series of measurements. In similar study statistically significant differences were identified in the vertical jump test from the fourth test session in relation to the first session, while statistically significant difference for standing long jump test was only found in the final sixth session (Vrbik et al., 2016). In both studies it took more than three sessions for significantly better tests results. In our study three sessions was sufficient to make significant better results in leg power tests during second measurements: Countermovement jump test (16.4\%) and Countermovement jump with free arms test $(22 \%)$. In the first and second measure there was only one attempt without any information about score and proper technique. Between them there were three sessions with three attempts providing familiarization and feedback information. With the introduction to the test, familiarization, it was attempted to remove the effect of learning. It seems that sufficient amount of information about the motor task make significantly better tests results in second measurement. There was also significantly better results in the Curl-up (60\%), Running speed (15\%), and Agility (5\%).

The efficacy of complex motor movement performance and the quickness of new motor structure acquisition depend on coordination, so only good quality movement and sufficient number of repetitions can improve this process.

In such circumstances, it is very important to provide children with additional instructions on the movement itself (Bala, 1999; Sullivan et al., 2008; Tomac et al., 2012). Tsigilis and Theodosiou (2008) attempt to establish whether the testing results remain stable across several attempts, or if they will change due to the influence of movement repetition. Their results are in accordance with several other studies (Ageberg, Zatterstrom, \& Moritz, 1998; Etnyre \& Poindexer, 1995; Ploutz-Snyder \& Giamis, 2001) in which a positive impact of repeated movements on result improvement was proven.

Results of the studies mentioned indicate that additional attempts and instructions can influence the process of motor learning. To optimize motor learning, children may require longer periods of practice.

In our study we hypothesized that short periods of treatment will prevent the impact of biological development and learning effect on motor tasks realization, and tests results improvement does not necessary represent changes in subjects performance, but it may reflect familiarization with the test. Our study also indicates that repeated movements and additional instruction about proper movement mechanics (which consist of 3 attempts every third day) affect the results of the tests with significantly better results in the final measurement when estimating explosive leg power, shoulder muscle repetitive strength, speed and agility. 
Some earlier research shows that the process of familiarization is necessary for the tests of maximal isotonic strength of knee extensor muscles (PloutzSnyder \& Giamis, 2001) and for the Wingate anaerobic test (Barfield, Sells, Rowe, \& Hanningan-Downs, 2002). On the other hand, Arteaga, Dorado, Chavarren, and Calbert (2000) indicate that it is not necessary to familiarize the subjects with the testing procedures for estimating jumping ability, more specifically the squat jumps and countermovement jumps. The authors tested active adult persons, basing their conclusions on the jump height recorded during six separate testing sessions. In our study, the subjects had significantly better results in the final measurement precisely in countermovement jumps with free arms, followed by countermovement jumps with fixed arms.

In another study, Minetti (2002) suggested that increase of force applied during the jump is affected by the decrease of time spent in contact with the ground and the increase of movement power and speed. Although the previous two studies did not include children, it appears that the subjects in our study have improved their jumping skills based on better understanding of jumping mechanics and better use of eccentric-concentric contraction and arm swing. During the three sessions of familiarization subject were given instruction to decrease time spent in contact with the ground, and how to use their arms during the jump, thus scoring better in the final measurement.

The familiarization has no impact on squat jump results, movement with only concentric contraction component, where improvement was only $4 \%$. This movement depends on strength applied in one direction which was not significantly improves in second measurement.

Moir et al. (2004) have attempted to establish the impact of familiarization on the reliability of results of jumping tests and running speed of physically active adults. Their results indicate a high reliability of test results in vertical jumping, as well as in 10-and 20-meter running speed tests, where the best result was recorded after three repetitions and did not change significantly under the influence of familiarization. The authors explain the lack of importance of familiarization by the status of the subject. In their study all subjects in that sample participated in sporting activities that contain jumps and sprints, and during measurements subjects showed competence in motor skills necessary for these tests and that is why additional sessions for mastering the movements were not necessary. It has been agreed (Bala, 1999; Sullivan et al., 2008) that it is not appropriate to apply adult protocols for children, and it is important to provide them with preparation and additional instructions. Tomac et al. (2012) have attempted to establish the impact of familiarization on standing long jump test results in 6-year-old children. Their results indicate that a period of familiarization with the motor task significantly improved their scores after the experimental treatment. In our study, as well as in the previous ones, subjects did not have experience with this kind of movement and it seems that additional information linked to appropriate jump mechanics make significant improvements in jump height in second measurement. Also, additional information of running mechanics (which include step frequency and length, pace, body position during turnovers) make significant improvements in speed and agility test results in the second measurement. It seems that familiarization, consisting of three sessions of three attempts each, is sufficient to affect results of these tests. During that period familiarization had a positive impact on jumping and running mechanics which lead to higher jump and running speed and better change of direction in agility tests.

With regard to the test of estimated abdominal muscle repetitive strength, it appears that familiarization had an impact on improvement of technique execution, which requires lifting the torso off the ground by 30 degrees, which was less than in the first attempt, and has thus facilitated the execution. According to the Shapiro-Wilks test, distribution does not deviate significantly from the normal distribution. The ICC coefficient between the series of measurements was found to be good .644, and has fair to high test-retest reliability (from .389. to .845). The main limitation of this study is the absence of a control group in order to distinguish the effect of repeated measurements and the effect of familiarization. This study was limited by a small number of examinees, so further research should include more participants and a control group. Nevertheless, the results indicate that the familiarization with movement mechanics played a significant role in results improvements. To avoid errors in motor skills assessment, a child should be prepared for motor task performance and for the situation in which the task is performed.

\section{Conclusions}

Results of this study indicate that familiarization with the test procedures prior to testing affect results of the motor tests. Subject significantly improved results for the assessment of leg power, abdominal repetitive strength, running speed and agility in the final measurement. It seems that feedback information and adequate instructions increase children's ability to better understand movement mechanics in mentioned motor skills. With regard to this, it was necessary to provide a sufficient amount of information about the motor task. This 
study indicates that familiarization is necessary during preschool children's motor skills testing in order for the results to be as precise as possible. Just as in the previous cases, this study has confirmed that the process of familiarization of preschool children with the testing procedures, as well as the feedback information about result and quality of technique during test, is an important segment of preschool children's motor skills evaluation.

\section{Conflict of interest}

There were no conflicts of interest.

\section{References}

Ageberg, E., Zatterstrom, R., \& Moritz, U. (1998). Stabilometry and one-leg hop test have high test-retest reliability. Scandinavian Journal of Medicine and Science in Sports, 8, 198-202.

American Academy of Pediatrics. (1992). Fitness, activity, and sports participation in the preschool child. Pediatrics, 90, 1002-1004.

American College of Sports Medicine. (2010). ACSM's Guidelines for Exercise Testing and Prescription (8th ed.). Philadelphia, PA: Lippincott Williams \& Wilkins.

Arteaga, R., Dorado, C., Chavarren, J., \& Calbert, J. A. L. (2000). Reliability of jumping performance in active men and women under different stretch loading conditions. Journal of Sports Medicine and Physical Fitness, 6, 26-34.

Bala, G. (1999). Some problems and suggestions in measuring motor behavior of pre-school children. Kinesiologia Slovenica, 5(2), 5-10.

Barfield, J. P., Sells, P. D., Rowe, D. A., \& Hanningan-Downs, K. (2002). Practice effect of the Wingate anaerobic test. Journal of Strength and Conditioning Research, 16, 472-473.

Barnett, L. M., van Beurden, E., Morgan, P. J., Brooks, L. O., \& Beard, J. R. (2008). Does childhood motor skill proficiency predict adolescent fitness? Medicine \& Science in Sports \& Exercise, 40, 2137-2144.

Burton, A. W., \& Miller, D. E. (1998). Movement skill assessment. Champaign, IL: Human Kinetics.

Bushnell, E. W., \& Boudreau, J. P. (1993). Motor development and the mind: The potential role of motor abilities as a determinant of aspects of perceptual development. Child Development, 64, 1005-1021.

Council of Europe. (1988). EUROFIT: Handbook for the EUROFIT tests of physical fitness. Rome, Italy: Author.

Eston, R., \& Reilly, T. (Eds.). (2001). Kinanthropometry and exercise physiology laboratory manual (2nd ed.). London, United Kingdom: Routledge.

Etnyre, B. R., \& Poindexter, H. B. W. (1995). Characteristics of motor performance, learning, warm-up decrement, and reminiscence during a balancing task. Perceptual Motor Skills, 80, 1027-1030.

Fisher, A., Reilly, J. J., Kelly, L. A., Montgomery, C., Williamson, A., Paton, J. Y., \& Grant, S. (2005). Fundamental movement skills and habitual physical activity in young children. Medicine \& Science in Sports \& Exercise, 37, 684-688.
Glaister, M., Witmer, C., Clarke, D. W., Guers, J. J., Heller, J. L., \& Moir, G. L. (2010). Familiarization, reliability, and evaluation of a multiple sprint running test using selfselected recovery periods. Journal of Strength and Conditioning Research, 24, 3296-3301.

Gwynne, K., \& Blick, B. (2004). Motor performance checklist for 5-year-olds: A tool for identifying children at risk of developmental co-ordination disorder. Journal of Paediatrics Child Health, 40, 369-373.

Hopkins, W. G. (2000). Measures of reliability in sports medicine and science. Sports Medicine, 30, 1-15.

Malina, R. M., \& Bouchard, C. (1991). Growth, maturation, and physical activity. Champaign, IL: Human Kinetics.

Minetti, A. E. (2002). On the mechanical power of joint extensions as affected by the change in muscle force (or cross-sectional area), ceteris paribus. European Journal of Applied Physiology, 86, 363-369.

Moir, G., Button, C., Glaister, M., \& Stone, M. H. (2004). Influence of familiarization on the reliability of vertical jump and acceleration sprinting performance in physically active men. Journal of Strength and Conditioning Research, 18, 276-280.

Moir, G., Sanders, R., Button, C., \& Glaister, M. (2005). The influence of familiarization on the reliability of force variables measured during unloaded and loaded vertical jumps. Journal of Strength and Conditioning Research, 19, 140-145.

Patterson, P., Bennington, J., \& De La Rosa, T. (2001). Psychometrics properties of child- and teacher-reported curl-up scores in children ages 10-12 years. Research Quarterly for Exercise and Sport, 72, 117-124.

Piek, J. P., Dawson, L., Smith, L. M., \& Gasson N. (2008). The role of early fine and gross motor development on later motor and cognitive ability. Human Movement Science, 27, 668-681.

Ploutz-Snyder, L., \& Giamis, E. (2001). Orientation and familiarization of 1RM strength testing in old and young women. Journal of Strength and Conditioning Research, 1, 519-523.

Sullivan, K. J., Kantak, S. S., \& Burtner, P. A. (2008). Motor learning in children: Feedback effects on skill acquisition. Physical Therapy, 88, 720-732.

Thomas, J. R., \& Nelson, J. K. (1996). Research methods in physical activity (3rd ed.). Champaign, IL: Human Kinetics.

Tomac, Z., \& Hraski, Z. (2016). Influence of familiarization of preschool children with motor tests on test results and reliability coefficients. Perceptual and Motor Skills, 123, 717-736.

Tomac, Z., Hraski, Z., \& Sporis, G. (2012). The assessment of preschool children's motor skills after familiarization with motor tests. Journal of Strength and Conditioning Research, 26, 1792-1798.

Tsigilis, N., \& Theodosiou, A. (2008). The influence of multiple administration of a psychomotor test on performance and learning. Journal of Strength and Conditioning Research, 22, 1964-1968.

Vrbik, I., Sporiš, G., Štefan, L., Madić, D., Trajković, N., Valantine, I., \& Milanović, Z. (2016). The influence of familiarization on physical fitness test results in primary school-aged children. Pediatric Exercise Science, 29, 278-284.

Yoon, D. Y., Scott, K., Hill, M. N., Levitt, N. S., \& Lambert, E. V. (2006). Review of three tests of motor proficiency in children. Perceptual Motor Skills, 102, 543-551. 